\title{
Programmed cell death protein-1/programmed death-ligand 1 blockade enhances the antitumor efficacy of adoptive cell therapy against non-small cell lung cancer
}

\author{
Jingyi Chen", Yusong Chen", Fenglan Feng", Cheng Chen, Haikang Zeng, Shuai Wen, Xin Xu, \\ Jianxing $\mathrm{He}^{\#}$, Jin $\mathrm{Li}^{\#}$
}

State Key Laboratory of Respiratory Disease, the Thoracic Surgery Department, the First Affiliated Hospital of Guangzhou Medical University, National Clinical Research Center for Respiratory Disease, Guangzhou 510120, China

Contributions: (I) Conception and design: J Li, J He; (II) Collection and assembly of data: Y Chen, J Chen, F Feng; (III) Data analysis and interpretation: Y Chen, J Chen; (IV) Manuscript writing: Y Chen, J Chen; (V) Collection of specimens: Xu Xin; (VI) Final approval of manuscript: All authors.

"These authors contributed equally to this work.

Correspondence to: Jin Li. State Key Laboratory of Pulmonary Diseases, 1st Affiliated Hospital of Guangzhou Medical University, No. 151, Yanjiang Rd, Guangzhou 510120, China. Email: lij1250@hotmail.com; Jianxing He. State Key Laboratory of Pulmonary Diseases, 1st Affiliated Hospital of Guangzhou Medical University, No. 151, Yanjiang Rd, Guangzhou 510120, China. Email: drhe_jianxing@163.com.

Background: Cytokine-induced killer (CIK) cells and natural killer (NK) cells are employed by two different approaches to adoptive cell immunotherapy for cancer. It has been reported that adoptive cell immunotherapy could prolong the overall survival (OS) of advanced cancer patients. The introduction of agents that induce immune checkpoint blockades has improved the efficacy of immune-mediated therapy for metastatic cancers. However, the effects of combining a checkpoint inhibitor with CIK cells or NK cells to target non-small cell lung cancer (NSCLC)remain unknown.

Methods: The present study investigated the effects of combining CIK cells with a programmed cell death protein-1 (PD-1) inhibitor (an anti-PD-1 monoclonal antibody). During the expansion cultivation, the addition of the PD-1 antibody promoted CIK-mediated cytotoxicity in H1975 lung adenocarcinoma cells. Co-cultivation of CIK cells with the PD-1 antibody for 6 days induced $\mathrm{CD}^{+} \mathrm{CD} 56^{+} \mathrm{T}$ cell expansion, with increases in the levels of CD107a and interferon $\gamma(\mathrm{IFN}-\gamma)$.

Results: When NK cells were co-cultured with $5 \mu \mathrm{g} / \mathrm{mL}$ of an anti-programmed death-ligand 1 (PD-L1) mAb for 24 hours at an effector cell: target ratio of 10:1, it led to more potent cytotoxicity compared to other time points and concentrations. However, combining NK cells with the anti-PD-L1 mAb showed no significant advantages over treatment with NK cells alone.

Conclusions: Our results suggest that combining CIK cells with PD-1 blockade before transfusion might improve the efficiency of CIK therapy for NSCLC patients. This effect does not seem to occur for NK cell therapy.

Keywords: Non-small cell lung cancer (NSCLC); cytokine-induced killer (CIK); natural killer (NK); programmed cell death protein-1 (PD-1); programmed death-ligand 1 (PD-L1)

Submitted Jul 05, 2018. Accepted for publication Oct 24, 2018.

doi: $10.21037 /$ jtd.2018.10.111

View this article at: http://dx.doi.org/10.21037/jtd.2018.10.111 


\section{Introduction}

Lung cancer is the most common cancer worldwide (1). Non-small cell lung cancer (NSCLC) accounts for about $85 \%$ of the lung carcinoma cases all over the world, and despite active treatments like surgical resection, targeted therapy, and adjuvant therapies, the 5 -year overall survival (OS) rate is approximately $15 \%$, which is lower than the survival rate for colon, breast, or prostate cancer $(1,2)$. A recently developed tumor immunotherapy technique, adoptive immunotherapy (AI), has shown beneficial clinical outcomes (3). Recent clinical success has emphasized the potential of using immunotherapy based on the adoptive cell transfer (ACT) of gene-engineered or naturally developing $\mathrm{T}$ cells to mediate tumor eradication in patients (4). $\mathrm{T}$ and NK lymphocytes are understood to be the primary effector cells of the immune system (5).

Two novel strategies are currently considered to be ideal candidate adjuvant therapies for cancer immunotherapy. One employs cytokine-induced killer (CIK) cells, which are CD3/CD56 double-positive T cells activated by a variety of cytokines in vitro. These cells exhibit a wide range of major histocompatibility complex (MHC)-unrestricted antitumor activity with confirmed survival benefits for hematological and advanced tumors (6-8). CIK cells can be generated from $80-100 \mathrm{~mL}$ of whole peripheral blood, followed by sequential incubation with peripheral blood mononuclear cells (PBMCs) in the presence of interferon $\gamma(\mathrm{IFN}-\gamma)$, an anti-CD3 monoclonal antibody (OKT3) and IL2 $(9,10)$. For each expansion, more than $1 \times 10^{10} \mathrm{CIK}$ cells are obtained for clinical infusion.

Using natural killer (NK) cells-based therapy is also considered to be an ideal candidate immunotherapy for cancers. NK cells can be generated from $30 \mathrm{~mL}$ of whole peripheral blood, followed by incubation with anti-human CD16 mAb and rhIL-2 plus irradiated (25 Gy) K562 feeder cells (in co-culture). The final NK cell population reaches $1 \times 10^{9}$ before infusion.

Recently, studies of immune checkpoints have been attracting increasing attention. The programmed cell death protein-1 (PD-1) receptor and its ligand, programmed death-ligand 1 (PD-L1), are involved in several types of cancers. The interaction of the PD-L1 expressed on cancer cells with PD-1 on the surface of T-cells results in an immune escape pathway that functions by preventing the activation of cytotoxic T-cells in the lymph nodes and their subsequent recruitment to the tumor (11). It has been hypothesized that combining CIK cells with PD-1 blockade would block the PD-1 receptor on tumor cells, resulting in more potent CIK cells because the immune escape system would be inhibited (12). Pre-culturing NK cells with a PDL1 inhibitor, a fully human IgG1 monoclonal antibody (mAb), would block the PD-1/PD-L1 pathway and might induce antibody-dependent cell-mediated cytotoxicity (ADCC) (13-16). The use of a PD-L1 inhibitor might also increase $\mathrm{ADCC}$, because the NK cells bind to the $\mathrm{Fc}$ region of the antibody via CD16 (FcyRIII) thereby killing tumor cells due to the release of perforins and granzymes $(15,17)$. Khanna et al. demonstrated that malignant mesothelioma (MM) cells highly express PD-L1 and are susceptible to ADCC by an anti-PD-L1 antibody (17). Although many tactics have provided exciting preclinical data, several difficulties in clinical translation have limited their therapeutic application to a fraction patient (18). The precise mechanism(s) underlying the tumor-killing in response to treatment with a combination of an immune checkpoint inhibitor with CIK cells or NK cells have not been completely elucidated. Thus, the present study in NSCLC examined the effects of co-incubating CIK cells and NK cells with a PD-1/PDL-1 blocker using a series of different concentrations and time points to identify the optimal approach and to clarify the mechanism(s) of action.

\section{Methods}

\section{Generation of CIK cells and co-incubation with PD-1 mAb}

CIK cells were generated from the PBMCs of healthy donors. In brief, the PBMCs were obtained from buffer coats of PBMCs using Human Lymphocyte Separation Medium (DAKEWE) and were washed three times with phosphate buffered saline (PBS). Next, the PBMCs were re-suspended at $1 \times 10^{6}$ cells/mL in GT-T551 H3 (TaKaRa) containing self-sera, and were stimulated with recombinant human IFN- $\gamma(1,000 \mathrm{U} / \mathrm{mL}$, T\&L Biological, Beijing, China) for 24 hours. The cells were then transferred to anti-CD3 (T\&L Biological, Beijing, China) pre-coated tissue-culture flasks, and stimulated with $500 \mathrm{IU} / \mathrm{mL}$ recombinant human interleukin-2 (125Ala) at $500 \mathrm{U} / \mathrm{mL}$ (SL-PHAM, Beijing, China) every 3 days until cells were harvested on day 12. These CIK cells were then cultured with a monoclonal PD-1 antibody (Bio-Thera Solutions Ltd., China) at a series of concentrations and time points as shown in the Supplementary data. On day 6 (G1-3), 10 (G4-6), or 11 (G7-9), the PD-1 monoclonal antibody was added at a final concentration of 1,2 , or $4 \mu \mathrm{g} / \mathrm{mL} / 10^{6}$ cells. 


\section{NK cell expansion and co-culture with PD-L1 mAb}

PBMCs were isolated from healthy donor peripheral whole blood using Ficoll (DAKEWE, CN). On day 0, the PBMCs were seeded at $1 \times 10^{6}$ cells $/ \mathrm{mL}$ and cultured with irradiated (25 Gy) K562 feeder cells $\left(10^{7}\right.$ cells $\left./ \mathrm{mL}\right)$ in $1 \mu \mathrm{g} / \mathrm{mL}$ anti-human CD16 mAb (eBioscience, San Diego, CA, USA)-coated culture plates. The NK cells and feeder cells were cultured in Roswell Park Memorial Institute (RPMI) 1640 supplemented with $5 \%$ human serum, L-glutamine, and IL-2 $(100 \mathrm{U} / \mathrm{mL})$ at $37{ }^{\circ} \mathrm{C}$ in a $5 \% \mathrm{CO}_{2}$ incubator. NK cells were harvested and cultured with a PD-L1 monoclonal antibody (T\&L Biological, Beijing, China) at a series of concentrations and time points as shown in the Supplementary data. On day 11 (G1-3), 12 (G4-6), or 13 (G7-9), $1 \times 10^{7}$ NK cells were cultured with the PD-L1 antibody at a final concentration of $1,2.5$, or $5 \mu \mathrm{g} / \mathrm{mL}$ in $10 \mathrm{~mL}$ medium.

\section{Cell lines}

The human lung adenocarcinoma cancer cell lines A549, H1299, SPC-A-1, and H1975, were maintained in DMEM medium (GIBCO) supplemented with 10\% FBS (GIBCO), which is hereafter called 'complete medium'.

\section{Degranulation assay (CD107a)}

CIK cells (cultured with or without the PD-1 antibody) and H1975 cells were plated at an effector: target (E: T) ratio of 10:1, 20:1, and incubated for 24 hours at $37^{\circ} \mathrm{C}$ in the presence of a CD107a-FITC mAb (BioLegend, San Diego, CA, USA). CIK cells degranulation was assessed by cell surface staining for the lysosomal marker CD107a by flowcytometry.

\section{Enzyme-linked immunosorbent assay (ELISA)}

To investigate the level of IFN- $\gamma$ (Elabscience) in the supernatants of H1975 lung cancer cells treated with CIK alone or in combination with the PD-1 mAb, an ELISA assay was performed according to the manufacturer's instructions. Briefly, approximately $1 \times 10^{5}$ cells treated with CIK were seeded in 96-well plates. The plates were incubated in a $5 \%$ humidified incubator at $37{ }^{\circ} \mathrm{C}$ for $24 \mathrm{~h}$. The cell supernatants were then collected to detect the concentration of IFN- $\gamma$.

\section{Lactate dehydrogenase (LDH) assay}

We performed the LDH release assay using the CytoToxONE $^{\text {TM }}$ Homogeneous Membrane Integrity Assay kit (Promega) to assess the cytotoxic activity of the CIK and NK cells. The effector CIK or NK cells for each treatment group were respectively cultured with $5 \times 10^{3} \mathrm{H} 1975$ tumor cells/well in triplicate at two different effectors: target ratios (20:1 and 10:1) and incubated at $37^{\circ} \mathrm{C}$ for 4 to 6 hours. The percent cytotoxicity was calculated according to the formula:

Percent cytotoxicity $=100 \times($ experimental - culture medium background)/(maximum LDH release - culture medium background)

\section{Real-time polymerase chain reaction (RT-PCR) assay}

Assessments of the mRNA expression levels in each group were performed as follows: total cellular RNA was isolated using the Trizol RNA isolation reagent according to the manufacturer's instructions (SigmaAldrich, St. Louis, MO, USA). A reverse transcription system (TAKARA) was used to generate first-strand template cDNA from $5 \mu \mathrm{g}$ of total RNA. The PCR reaction was then performed as follows: denaturation at $95{ }^{\circ} \mathrm{C}$ for $5 \mathrm{~min}$, followed by 40 cycles of $95{ }^{\circ} \mathrm{C}$ for $5 \mathrm{~s}$, $60{ }^{\circ} \mathrm{C}$ for $60 \mathrm{~s}$, and $72{ }^{\circ} \mathrm{C}$ for $30 \mathrm{~s}$. SYBR-Green Q-PCR Master Mix was used according to the manufacturer's instructions (KAPA, USA). The sequences of primers were as follows:

(I) PD-L1: 5'-TGCCGACTACAAGCGAATTACTG3'(sense) and 5'-CTGCTTGTCCAGATGACTTC GG-3'(antisense);

(II) beta-actin: 5'-CACAGAGCCTCGCCTTTGC-3' (sense) and 5'-GCGCGGCGATATCATCATCC-3' (antisense).

The expression of beta-actin was considered as a reference gene and served as a control for the cDNA integrity.

\section{Antibodies}

The following anti-human antibodies were used according to the manufacturer's instructions: CD3-PerCP, CD4FITC, CD8-PE, CD16-PE, and CD56-PC7 from BD Biosciences (San Jose, CA, USA) and PD-L1-PC-5, natural killer group 2D (NKG2D)-PE-CY5, and CD107a-FITC from BioLegend. 


\section{Immunofluorescent staining (IFC)}

The cells were fixed in $100 \%$ methanol $(10 \mathrm{~min})$, and blocked with $10 \% \mathrm{BSA} / 0.2 \% \mathrm{PBS}$-Tween for $30 \mathrm{~min}$ at $37{ }^{\circ} \mathrm{C}$. They were then incubated with the anti-PD-L1 antibody (ab205921; Abcam) used at a 1/100 dilution overnight at $4{ }^{\circ} \mathrm{C}$. The secondary antibody (pseudo-colored green) was Alexa Fluor ${ }^{\circledR} 488$ goat anti-Rabbit IgG (Life Technologies Corporation), which was used at a $1 / 1,000$ dilution for a 45 min incubation at room temperature. ProLongR Gold Antifade Reagent with DAPI (Life Technologies, Carlsbad, USA) was used to stain the cell nuclei (pseudo-colored blue).

\section{Models of tumor metastasis}

Human lung cancer cells were used as reference target cells, and were grown in DMEM medium supplemented with $10 \%$ fetal bovine serum, $100 \mathrm{U} / \mathrm{mL}$ penicillin and $100 \mathrm{mg} / \mathrm{mL}$ streptomycin. To develop the metastatic model, 3-4-week-old female nod/scid mice were transplanted with NSCLC cells $\left(1.0 \times 10^{6}\right.$ in $100 \mu \mathrm{L}$ of PBS) through the vein under sterile conditions (19), followed by an intravenous injection of CIK cells $\left(1.0 \times 10^{7}\right)$ on day 0 . On day 3 , the mice were again injected with CIK cells $\left(1.0 \times 10^{7}\right)$ intravenously via a tail vein. All of the mice were sacrificed by perfusion 14 days after the second injection, and their spleens, lungs, and livers were harvested. The lungs were then fixed and sectioned (3 $\mu \mathrm{m})$ in preparation for hematoxylin and eosin $(\mathrm{H} \& \mathrm{E})$ staining to determine the site(s) and multiplicity of metastasis.

\section{Results}

\section{In vitro expansion of effector cells derived from bealthy buman PBMCs}

The CIK cells were expanded as described in the Materials and Methods section. For our culture system, the CIK cells used for daily clinical infusion yield hundreds-fold changes compared to the original number of PBMCs. Representative data are shown in Figure $1 A$, which had a final cell count of $2.12 \times 10^{10}$. During cell culture, the proportions of cells that were positive for the T-cell marker $\left(\mathrm{CD}^{+}\right)$and the cytotoxic T-cell marker (CD8 $\left.{ }^{+}\right)$were D0, $18 \%$; D4, $55.33 \%$; and D12, 63.73\% (Figure 1B), The proportions of $\mathrm{CD}^{+}{ }^{+} \mathrm{CD} 4{ }^{+}$cells were D0, $30.05 \%$; D4, $40 \%$; and D12, $20 \%$, and the $\mathrm{CD}^{+}{ }^{+} \mathrm{CD} 56^{+}$cell ratios (D0, $1.78 \%$; D4, $9.79 \% ; \mathrm{D} 12,23.16 \%)$ were also significantly increased (Figure 1B). For experiments to investigate the effects of combining PD-1 blockade with CIK, $30 \mathrm{~mL}$ of fresh whole blood was collected, and CIK cells were generated from the PBMCs. After a 12-day incubation, $5.25 \times 10^{9} \mathrm{CIK}$ cells were harvested. The following percentages of cells were detected by flow cytometry on day 12 after routine expansion: $66.79 \% \mathrm{CD}^{+}{ }^{+} \mathrm{CD} 8^{+}, 24.51 \% \mathrm{CD}^{+} \mathrm{CD}^{+}$, and $22.7 \%$ $\mathrm{CD}^{+} \mathrm{CD}^{+} 6^{+}$(Figure 1C).

\section{Phenotypes of CIK cells obtained following anti-PD-1 treatment}

To determine the optimal timing and concentration of the PD-1 mAb, we examined the impact of a series of time points and concentrations on the final CIK cells generated. The time points used for the PD-1 blockade in CIK cell culture are shown in Figure S1. We found that after incubation of the CIK cells with the PD-1 mAb for 6 days at $1,2,4 \mu \mathrm{g} / \mathrm{mL} / 10^{6} \mathrm{CIK}$ cells, the percentages of $\mathrm{CD}^{+} \mathrm{CD}^{+}$cells were $24.51 \%(\mathrm{G} 1$, at $1 \mu \mathrm{g} / \mathrm{mL}), 24.79 \%$ (G2, at $2 \mu \mathrm{g} / \mathrm{mL})$, and $24.43 \%$ (G3, at $4 \mu \mathrm{g} / \mathrm{mL})$. When the cells were incubated for less than 48 hours, the percentages were $\mathrm{G} 4,35.64 \%$ at $1 \mu \mathrm{g} / \mathrm{mL}$; G5, $34.26 \%$ at $2 \mu \mathrm{g} / \mathrm{mL}$ and G6, $35.64 \%$ at $4 \mu \mathrm{g} / \mathrm{mL}$. Similarly, when cells were incubated for only 1 day, the percentages were: G7, 32.98\% at $1 \mu \mathrm{g} / \mathrm{mL} ; \mathrm{G} 8,34.66 \%$ at $2 \mu \mathrm{g} / \mathrm{mL}$ and $\mathrm{G} 9,31.76 \%$ at $4 \mu \mathrm{g} / \mathrm{mL}$ (Figure 1D).

When the cells were co-cultured with the mAb for 6 days, the percentages with the $\mathrm{CD}^{+} \mathrm{CD}^{+}$subtype were $66.79 \%$ (G1, at $1 \mu \mathrm{g} / \mathrm{mL}), 68.95 \%(\mathrm{G} 2$, at $2 \mu \mathrm{g} / \mathrm{mL})$, and $65.03 \%$ (G3, at $4 \mu \mathrm{g} / \mathrm{mL}$ ) which were not significantly different compared with the findings after incubation for 48 hours (G4, $61.91 \%$ at $1 \mu \mathrm{g} / \mathrm{mL} ; \mathrm{G} 5,60.96 \%$ at $2 \mu \mathrm{g} / \mathrm{mL}$; G6, $59.13 \%$ at $4 \mu \mathrm{g} / \mathrm{mL}$ ) or 24 hours $(\mathrm{G} 7,60.27 \%$ at $1 \mu \mathrm{g} / \mathrm{mL}$; $\mathrm{G} 8,61.49 \%$ at $2 \mu \mathrm{g} / \mathrm{mL}$; G9, $61.97 \% 4 \mu \mathrm{g} / \mathrm{mL}$ ) (Figure $1 E$ ).

The expression of the $\mathrm{CD}^{+} \mathrm{CD} 56^{+}$subgroups following a 6-day incubation with the $\mathrm{mAb}$ were $36.64 \%$ (G1, at $1 \mu \mathrm{g} / \mathrm{mL}), 33.61 \%(\mathrm{G} 2$, at $2 \mu \mathrm{g} / \mathrm{mL}$ ), and $33.71 \%$ (G3, at $4 \mu \mathrm{g} / \mathrm{mL}$ ) which were significantly higher than those following incubation for 48 hours (G4, 22.03\% at $1 \mu \mathrm{g} / \mathrm{mL}$; $\mathrm{G} 5,23.26 \%$ at $2 \mu \mathrm{g} / \mathrm{mL}$; G6, $23.19 \%$ at $4 \mu \mathrm{g} / \mathrm{mL}$ ) or 24 hours (G7, 21.91\% at $1 \mu \mathrm{g} / \mathrm{mL}$; G8, $21.64 \%$ at $2 \mu \mathrm{g} / \mathrm{mL}$; G9, $21.31 \% 4 \mu \mathrm{g} / \mathrm{mL}$ ) (Figure $1 F$ ). These results showed that the proportion of $\mathrm{CD}^{+} \mathrm{CD} 56^{+} \mathrm{CIK}$ cells was significantly higher in the groups co-cultured with the mAb for 6 days compared with the groups co-cultured for only 1 or 2 days.

We also examined whether the antibody affected the expression of PD-1 on CIK cells. We found no significant differences in the PD-1 expression on CIK cells among 
A

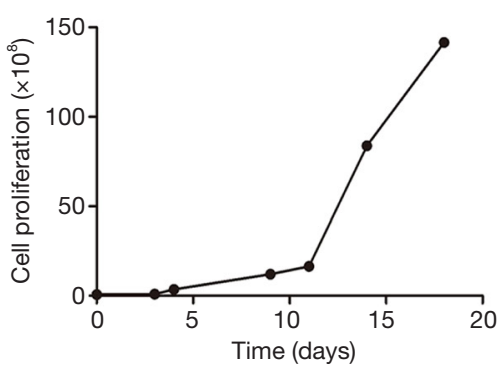

B

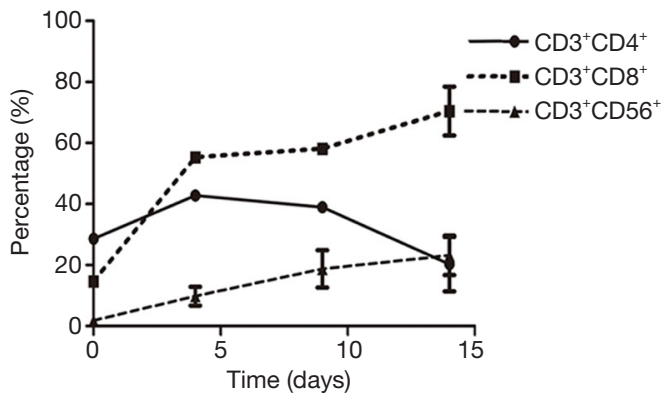

C
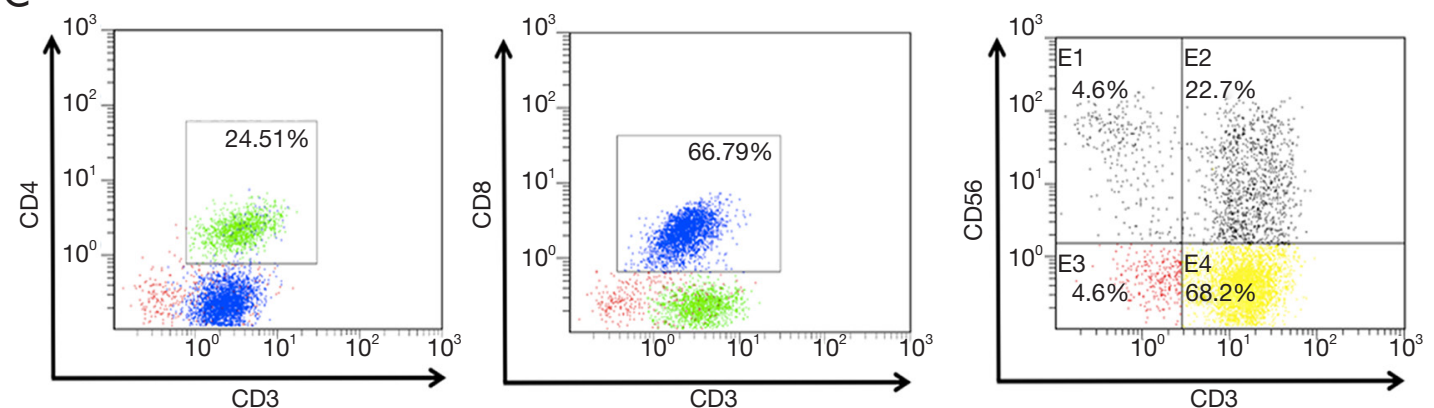

D

E
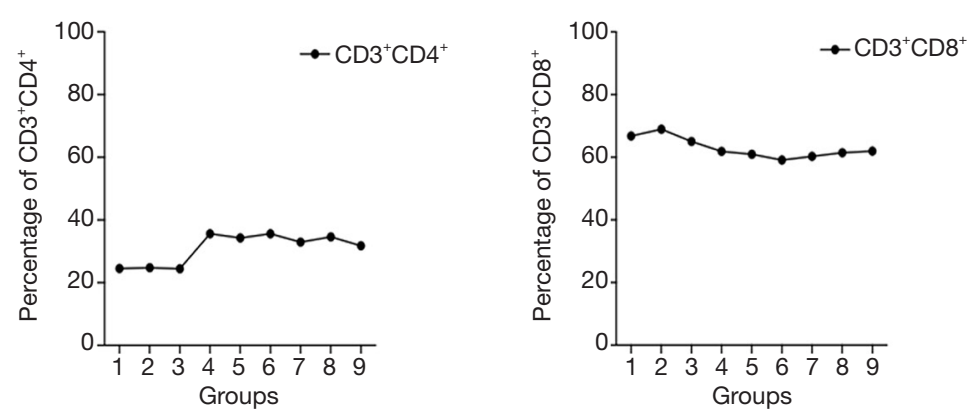

F

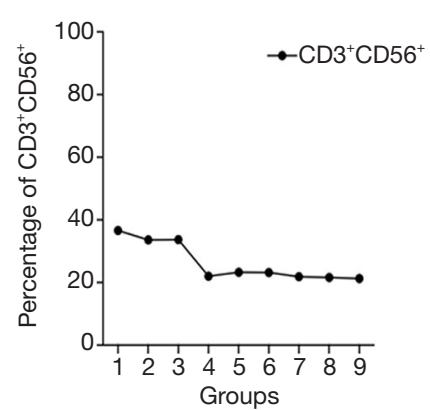

Figure 1 In vitro expansion and main phenotypes of CIK cells. CIK cells were obtained from fresh hPBMCs and expanded with anti-CD3 mAb and IFN- $\gamma$ plus rhIL-2 for 12 days (A). The $\mathrm{CD}^{+} \mathrm{CD}^{+}, \mathrm{CD}^{+} \mathrm{CD} 8^{+}$, and $\mathrm{CD}^{+} \mathrm{CD} 56^{+}$expression of cultured CIK was analyzed by flow cytometry (B,C). After treatment with anti-PD-1 antibody: G1-3 (1, 2, or $4 \mu \mathrm{g} / \mathrm{mL}$ for 6 days), G4-6 (1, 2, or $4 \mu \mathrm{g} / \mathrm{mL}$ for 48 hours), G7-9 $\left(1,2\right.$, or $4 \mu \mathrm{g} / \mathrm{mL}$ for 24 hours). The proportion of CIK cells of CD4 ${ }^{+}$subgroup, $\mathrm{CD}^{+}$subgroup and $\mathrm{CD}^{+} \mathrm{CD} 56^{+}$subgroup were shown in D, E and F respectively. CIK, cytokine-induced killer; hPBMCs, human peripheral blood mononuclear cells; IFN- $\gamma$, interferon $\gamma$; PD-1, programmed cell death protein-1.

different groups related to the anti-PD-1 treatment. This suggests that there is no stimulation or suppression of the receptor expression by the drug or the culture conditions used in our study.

\section{Screening for PD-L1 expression on NSCLC cell lines}

To determine the expression of PD-L1 in NSCLC cell lines, we first detected the $\mathrm{mRNA}$ level and protein expression of PDL1 using Q-PCR and IFC staining. As shown in Figure 2A,
PD-L1 mRNA was widely expressed by the NSCLC cell lines examined. Interestingly, the relative level of PD-L1 mRNA level on H1975 cells was 25.6-fold higher than that on A549 (0.9), SPC-A-1 (2.78), and H1299 cells (1.04). It was also expressed on normal human bronchial epithelial (HBE) cells. Flow cytometry (FCM) was subsequently used to examine the expression of PD-L1 on NSCLC cells. H1975 cells exhibited higher expression of the PD-L1 protein, with levels about 10-fold those in A549 and H1299 cells and about 200-fold that in the SPC-A-1 cells (Figure 2B), 
A
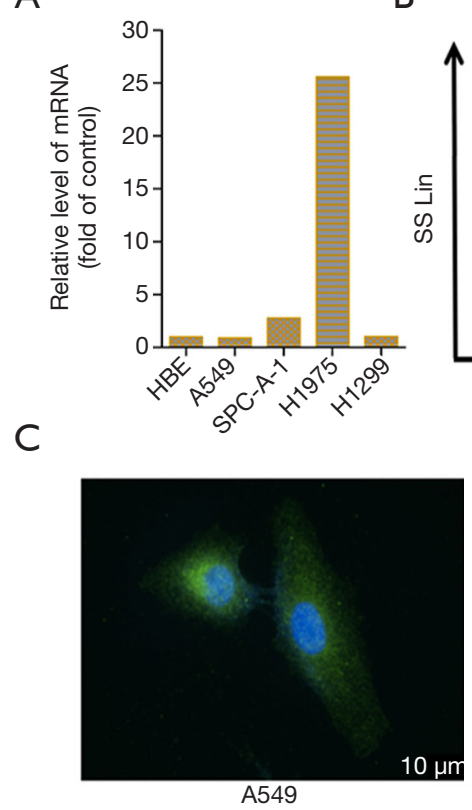

B
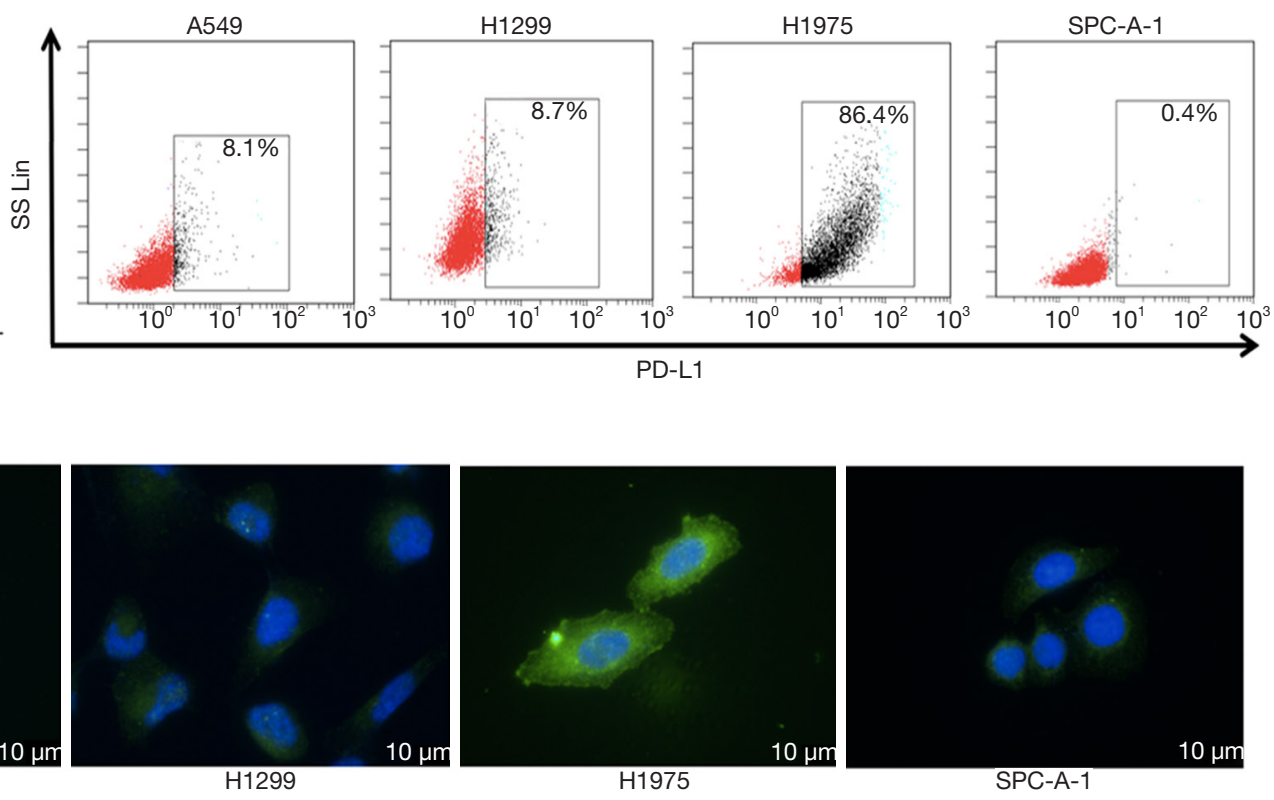

Figure 2 The expression of PD-L1 on NSCLC cell lines. PD-L1 was widely expressed on NSCLC cell lines, especially H1975 cells (A,B,C). PD-L1, programmed death-ligand 1; NSCLC, non-small cell lung cancer.

which was consistent with the Q-PCR results.

We next determined the distribution of the PD-L1 protein on these NSCLC cells by immunofluorescence (IFC). The IFC results showed that PD-L1 was expressed on the NSCLC cell membranes and in the cytoplasm (Figure 2C). The H1975 cells were selected as the optimal target tumor cells for subsequent assays due to their higher expression of PD-L1. These cells harbor the T790MEGFR mutation, but we have previously demonstrated that PD-L1 expression is not affected by EGFR mutation (20).

\section{Synergistic effects of combined treatment with CIK and a $P D-1$ inbibitor in vitro and in vivo}

To evaluate whether the PD-1 blockade could synergistically improve the cytotoxicity of $\mathrm{CD} 3^{+} \mathrm{CD} 56^{+} \mathrm{CIK}$ cells against NSCLC cells, we performed the LDH assay in vitro. As shown in Figure $3 \mathrm{~A}$, treatment with the PD-1 mAb enhanced the elimination of $\mathrm{H} 1975$ cells. While $8.84 \%$ and $15.13 \%$ of NSCLC cells were killed by CIK alone at E/T ratios of 10:1 and 20:1, respectively (Figure $3 A$ ), these values were $31.51 \%$ to $86.35 \%$ for the combined treatment with CIK cells with the PD-1 inhibitor. The IFN- $\gamma$ secretion showed no significant differences among the groups based on the LDH assay (Figure 3B).

Next, we examined the differences in cytokine secretion between CIK cells with and without PD-1 treatment when used to target H1975 cells. Compared with the control group (18\% of cells), $31.42 \%$ of cells were detected to have CD107a by the FCM assay when the CIK cells were used at an E/T ratio 20:1 along with PD-1 antibody at a concentration of $1 \mu \mathrm{g} / \mathrm{mL} / 10^{6} \mathrm{CIK}$ cells for 6 days. This was a 1.74-fold increase in CD107a expression (Figure 3C).

We further evaluated the therapeutic effects of using CIK cells combined with the PD-1 inhibitor in vivo. A total of $1 \times 10^{7} \mathrm{CIK}$ cells (in $100 \mu \mathrm{L}$ PBS) were co-cultured with or without PD-1 blockade then injected intravenously into mice bearing lung tumors on day 0 and day 3 (control = PBS only, each group: $\mathrm{n}=3$ ). We obtained a single cell suspension by grinding the mouse spleens, and it was found that there was enhancement of the proportion of $\mathrm{CD}^{+} \mathrm{CD} 56^{+}$cells (0.62\% vs. $0.09 \%)$, as well as CD107a expression (1.04\% vs. $0.5 \%$ ) in the CIK cells treated with the PD-1 inhibitor, as determined by the FCM analysis (Figure 3D). The mice treated with CIK cells with PD-1 blockade showed significantly better tumor growth inhibition compared with the PBS-treated control group, as shown in Figure 3E. Although treatment with CIK alone also reduced the tumor 
A

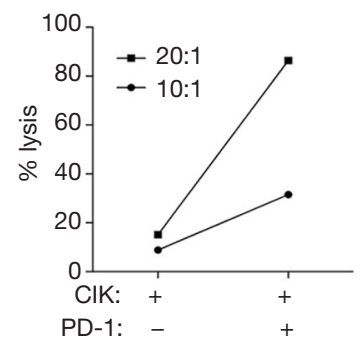

C

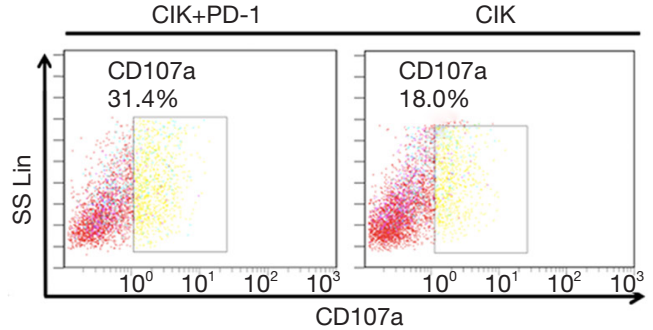

B

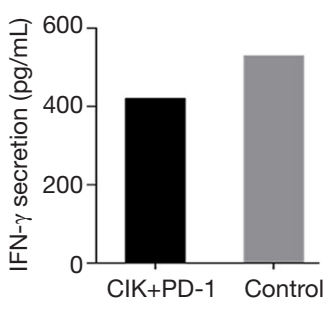

D

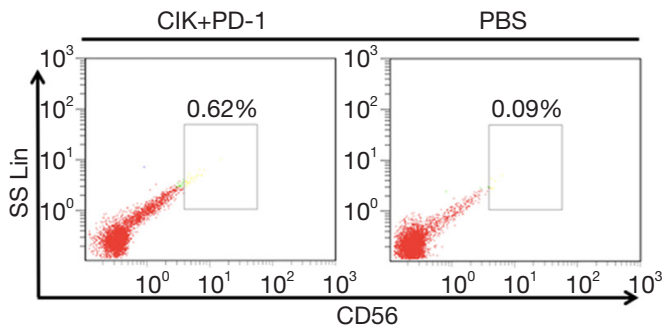

E
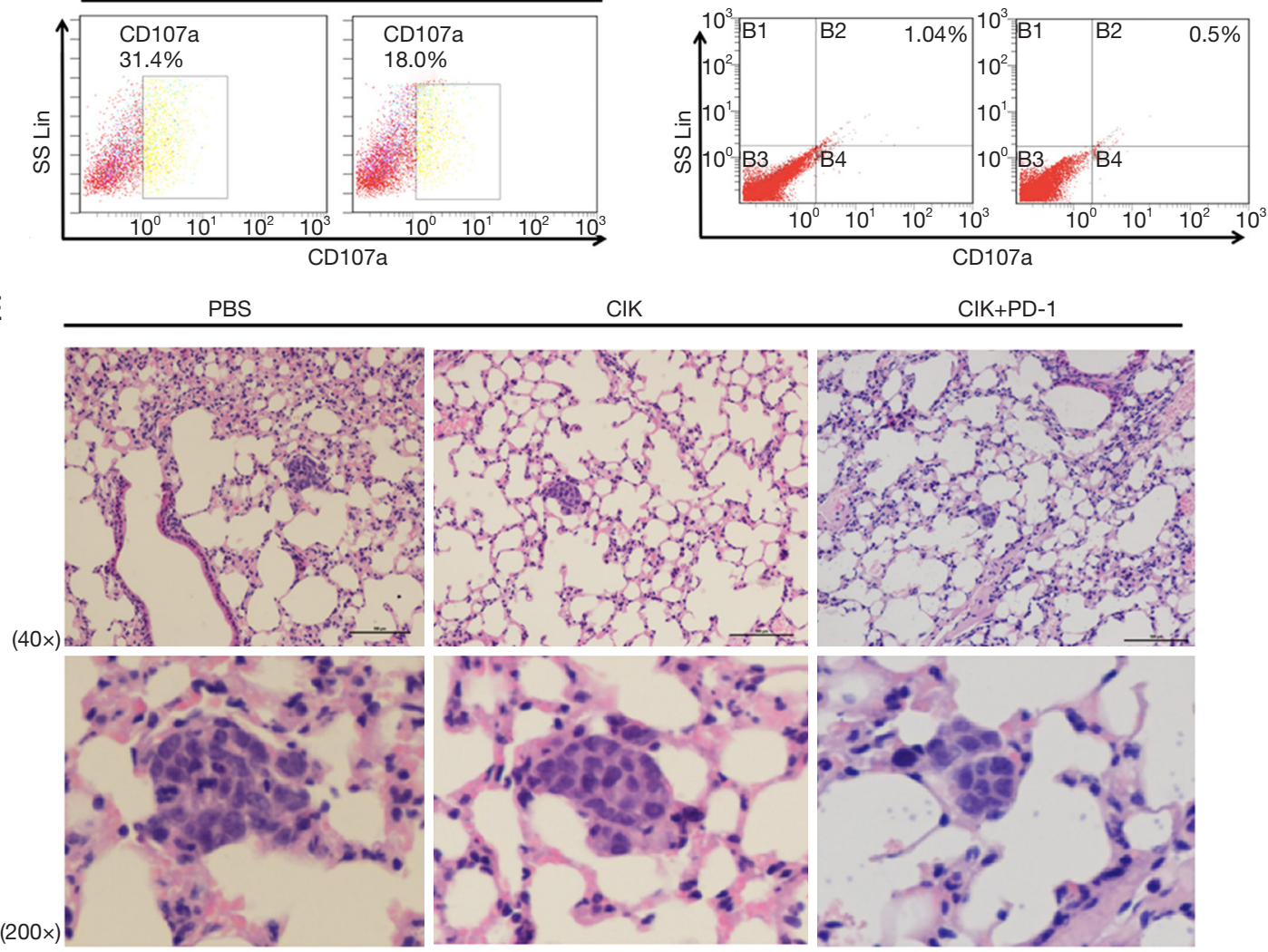

Figure 3 Effects of CIK cells treated with the PD-1 antibody in vitro and in vivo. The in vitro cytotoxicity of CIK cells (treated with or without the PD-1 mAb) against H1975 after 24 hours (A,B). The phenotypes of the CIK cells treated with the PD-1 antibody in comparison with CIK cells alone after co-culture with H1975 cells for 24 hours (as determined by FCM) (C). CIK cells cultured with the PD-1 antibody (at $1 \times 10^{7}$ cells/mouse) were intravenously administered via a tail vein on day 0 and day 3 . Single cells were obtained by grinding mouse spleens, and showed enhancement of the $\mathrm{CD} 3^{+} \mathrm{CD} 56^{+}$proportion, as well as $\mathrm{CD} 107 \mathrm{a}$ expression (D). The tumor nodules were measured after $\mathrm{H} \& \mathrm{E}$ staining to estimate tumor toxicity (E). CIK, cytokine-induced killer; PD-1, programmed cell death protein-1; FCM, flow cytometry.

volumes, the treatment was less effective.

\section{The alteration of CIK cells by PD-1 blockade}

To further investigate the mechanisms underlying the enhanced cytotoxicity of CIK cells following treatment with the PD-1 mAb, we performed an FCM assay to analyze the population of CIK cells (treated with or without PD-1 mAb) after co-culture with H1975 cells for 24 hours. We found that the phenotypic proportions of CIK cells were affected by pre-incubation with the PD-1 mAb. In total, $27.39 \%$ of cells were $\mathrm{CD}^{+}{ }^{+} \mathrm{CD} 4^{+}$and $65.51 \%$ were $\mathrm{CD} 3{ }^{+} \mathrm{CD} 8^{+}$after 
PD-1 blockade, values that were not significantly different from the CIK without PD-1 treatment $30.05 \%$ for $\mathrm{CD}^{+}{ }^{+} \mathrm{CD}^{+}$and $63.73 \%$ for $\mathrm{CD}^{+}{ }^{+} \mathrm{CD} 8^{+}$) (Figure $\mathrm{S} 2 \mathrm{~A}$ ). After PD-1 antibody treatment, the proportion of $\mathrm{CD} 56^{+}$cells in the $\mathrm{CD}^{+}$cell subset $(87.94 \%$ of cells) reached $29.9 \%$. The proportion of $\mathrm{CD}^{+} \mathrm{CIK}$ cells (without PD-1 blockade) was slightly higher at $91 \%$, however, the proportion of $\mathrm{CD} 56^{+}$ cells in this subset was only $22.7 \%$. This suggests that treatment with the PD-1 mAb altered the CIK cell subsets, enhancing the proportion of $\mathrm{CD}^{+} \mathrm{CD} 56^{+}$cells, which are the main effective and important components required to kill tumor cells. Moreover, the proportion of NK cells with NKG2D, which enables the elimination of tumor cells, was also higher in cells treated with this PD-1 blockade (14\%) than in control cells (6.4\%) (Figure S2B).

\section{Effects of NK cells with PD-L1 blockade}

As NKT cells were also affected by the above checkpoint inhibitor, we examined whether H1975 cells exhibited increased sensitivity to NK cells after treatment with a monoclonal antibody targeting PD-L1. We generated NK cells from $30 \mathrm{~mL}$ whole blood, and around $8 \times 10^{9} \mathrm{NK}$ cells were collected on Day 14. The different E/T ratios and timing of inhibitor treatment used in the experiments are shown in Figure $S 3$. The results of the LDH assay are presented in Figure 4A. The NK cells co-incubated with $5 \mu \mathrm{g} / \mathrm{mL}$ PD-L1 mAb for 24 hours showed the highest cytotoxicity to the H1975 cells. The inhibitor treatment increased the cytotoxicity to $100 \%(5 \mu \mathrm{g} / \mathrm{mL}, 24$ hours of PD-L1 inhibition, E/T ratio of 20:1) from $41 \%(5 \mu \mathrm{g} / \mathrm{mL}$, 72 hours of PD-L1 inhibition, E/T ratio of $20: 1$ ) and $1.7 \%$ ( $5 \mu \mathrm{g} / \mathrm{mL}, 72$ hours of PD-L1 inhibition, E/T ratio of 10:1). The weakest promotion of cytotoxicity $(0.3 \%)$ was observed in the group incubated with $1 \mu \mathrm{g} / \mathrm{mL}$ PD-L1 for 72 hours at an $\mathrm{E} / \mathrm{T}$ ratio of 10:1. However, even that combination treatment showed a slight enhancement of the cytotoxicity when compared to the blank control. Nevertheless, as shown in Figure 4B, there were no significant differences in the cytotoxicity between NK cells treated with or without the PD-L1 antibody for 24 hours.

To determine whether the PD-L1 antibody bound to NK cells via CD16 (FcyRIII), we analyzed the expression of $\mathrm{PD}-\mathrm{L} 1$ on NK cells (in the presence or absence of the PD$\mathrm{L} 1 \mathrm{mAb}$ ) using FCM. The results are shown in Figure $4 C$. PD-L1 was detected in the combination treatment group $(13.3 \%)$ at a higher level compared to the control (10.1\%). In addition, the ratios of the components of NK cells were affected when they were co-cultured with target tumor cells after pre-incubation with the PD-L1 mAb (Figure 4C). When the cells were incubated at an E/T ratio of 20:1, CD 107 a was expressed by $4.72 \%$ of cells in the combination group, which was 10 -fold higher than the $0.47 \%$ observed in the NK cells incubated without the antibody (Figure 4D). The NKG2D expression after PD-L1 treatment was not significantly different from that in the untreated cells $(45.8 \%$ vs. 41.6\%; Figure 4E).

\section{Discussion}

Immunotherapy has become a standard treatment for patients with certain cancers. Several PD-1 inhibitors have been developed, including pembrolizumab and nivolumab, and PD-L1 inhibitors atezolizumab, durvalumab, and avelumab are Food and Drug Administration (FDA)approved for the treatment of melanoma, lung cancer, colorectal cancer, kidney cancer and urothelial cancer, among others $(21,22)$. Our present study provides the first detailed examination to determine the most appropriate PD-1/PD-L1 concentration and duration of incubation with CIK cells and NK cells for use against NSCLC. We investigated the synergism between CIK cells and a PD$1 \mathrm{mAb}$ in vitro and in vivo. The results showed that coincubation of CIK cells with $1 \mu \mathrm{g} P D-1 \mathrm{mAb} / \mathrm{mL} / 10^{6} \mathrm{CIK}$ cells (for 6 days at an $\mathrm{E}$ : $\mathrm{T}=10: 1$ ) markedly promoted the CIK cells' cytotoxicity. Previous studies have shown that CIK cells possess dual properties and can exert activities like both $\mathrm{T}$ and NK cells $(7,23)$. The enhancement of the cytotoxicity by PD-1 might be associated with the alteration of CIK subsets (as N-like T cells). Based on our finding in CIK cells, we further investigated whether the anti-tumor activity of NK cells would also be promoted by PD-1/PD-L1 blockade. It was hypothesized that PD-L1 might be involved in the anti-tumor activity of NK cells through ADCC. Our study found that the strongest NK cell-mediated lysis occurred when they were co-cultured with a PD-L1 antibody at $5 \mu \mathrm{g} / \mathrm{mL} / 1 \times 10^{7}$ for 24 hours at an $\mathrm{E} / \mathrm{T}$ ratio of 20:1. However, the cytotoxicity of NK cells was not markedly enhanced by the PD-L1 mAb treatment compared to highest killing rate of NK cells alone. This might be because ADCC-mediated lysis is only one of the mechanisms underlying the anti-tumor activity of NK cells. Moreover, the anti-tumor effects of NK cells might be mainly dependent on the NKG2D pathway. For example, Ferrari de Andrade et al. reported that MICA and MICB are expressed on many cancer cells, and can tag cells to be 
A

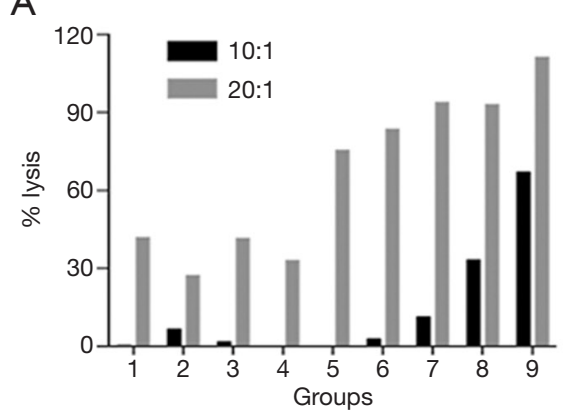

C

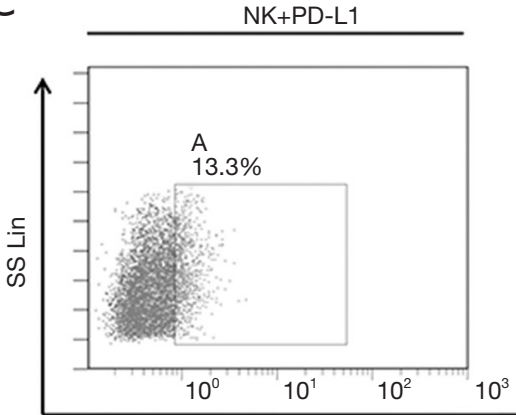

B
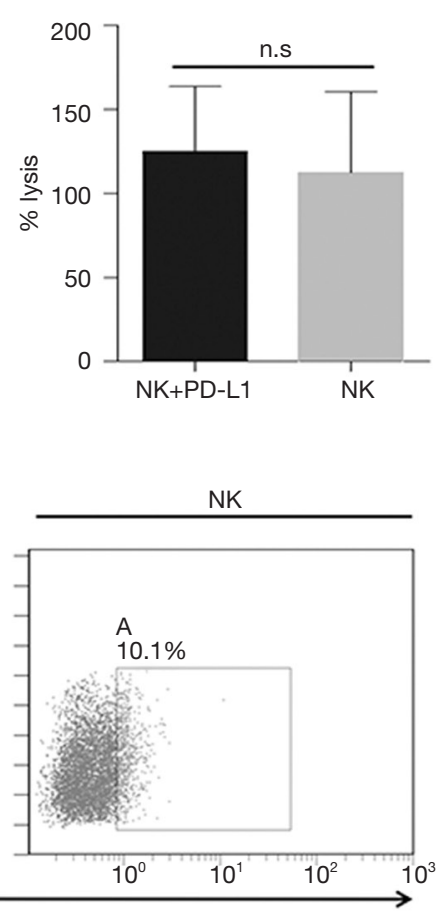

PD-L1

$\mathrm{D}$
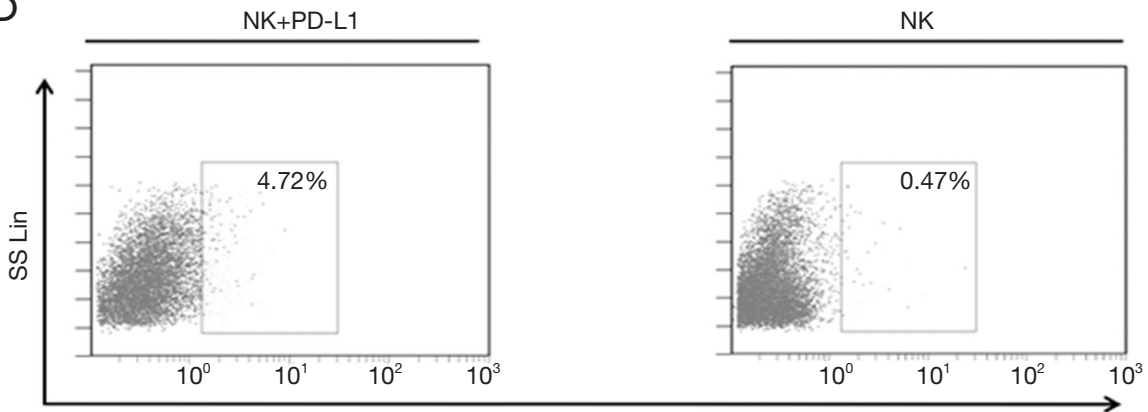

CD107a

E

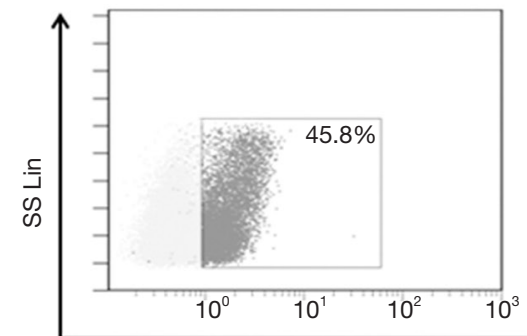

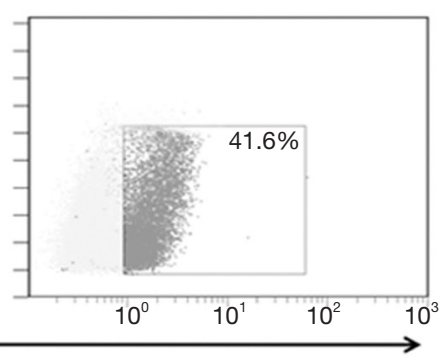

Figure 4 The effects of treatment with an anti-PD-L1 monoclonal antibody on NK cells. The PD-L1 antibody was used as follows: G1-3 (1, 2.5, or $5 \mu \mathrm{g} / \mathrm{mL}$ for 72 hours), G4-6 (1, 2.5, or $5 \mu \mathrm{g} / \mathrm{mL}$ for 48 hours), G7-9 (1, 2.5, or $5 \mu \mathrm{g} / \mathrm{mL}$ for 24 hours). The cells were then respectively co-cultured with H1975 cells at an E:T ratio of 10-20:1 and the extent of lysis was estimated by the LDH assay (A). The cytotoxicity of the combination treatment compared with treatment with NK cells alone (B). The phenotypic signatures of NK cells treated with the PD-L1 antibody for 24 hours at an E:T ratio of 20:1 against H1975 (analyzed by flow cytometry). PD-L1 (C), CD107a (D), NKG2D (E). PD-1, programmed cell death protein-1; NK, natural killer; LDH, lactate dehydrogenase; NKG2D, natural killer group 2D. 
eliminated by cytotoxic lymphocytes through NKG2D receptor activation (24). We observed that the NKG2D expression on NK cells was not altered following treatment with the PD-L1 mAb in this study. However, in our system, the total cytotoxicity of NK cells was higher than that of CIK combined with PD-1 in vitro, even without PD-L1 blockade. The effects of PD-1/PD-L1 blockade combined with cell therapy, for both T cells and NK cells together, needs further exploration in future studies. The results of the present study provide guidance on the optimal timing and concentrations to use for such studies, and for future clinical trials.

\section{Acknowledgements}

Funding: This study was supported in part by the National Natural Science Foundation of China (81672270) and Key project of Guangzhou Science Technology and Innovation committee (201707020042).

\section{Footnote}

Conflicts of Interest: The authors have no conflicts of interest to declare.

Ethical Statement: All human samples and data were obtained according to a protocol reviewed and approved by the local ethical committee, and all patients signed an informed consent form (2016-77).

\section{References}

1. Goldstraw P, Ball D, Jett JR, et al. Non-small-cell lung cancer. Lancet 2011;378:1727-40.

2. Nanavaty P, Alvarez MS, Alberts WM. Lung cancer screening: advantages, controversies, and applications. Cancer Control 2014;21:9-14.

3. Restifo NP, Dudley ME, Rosenberg SA. Adoptive immunotherapy for cancer: harnessing the $\mathrm{T}$ cell response. Nat Rev Immunol 2012;12:269-81.

4. Zeng Y, Ruan W, He J, et al. Adoptive Immunotherapy in Postoperative Non-Small-Cell Lung Cancer: A Systematic Review and Meta-Analysis. PLoS One 2016;11:e0162630.

5. Ruella M, Kalos M. Adoptive immunotherapy for cancer. Immunol Rev 2014;257:14-38.

6. Liu LW, Yang MY, Zhou M, et al. Improvement of cytotoxicity of autologous CIKs from patients with breast cancer to MCF-7 cells by suppressed PD-1 expression.
Cancer Biomark 2017;20:609-15.

7. Dai C, Lin F, Geng R, et al. Implication of combined PDL1/PD-1 blockade with cytokine-induced killer cells as a synergistic immunotherapy for gastrointestinal cancer. Oncotarget 2016;7:10332-44.

8. Gao X, Mi Y, Guo N, et al. Cytokine-Induced Killer Cells As Pharmacological Tools for Cancer Immunotherapy. Front Immunol 2017;8:774.

9. Introna $\mathrm{M}$. CIK as therapeutic agents against tumors. J Autoimmun 2017;85:32-44.

10. Introna $M$, Correnti F. Innovative Clinical Perspectives for CIK Cells in Cancer Patients. Int J Mol Sci 2018;19.

11. Robainas M, Otano R, Bueno S, et al. Understanding the role of PD-L1/PD1 pathway blockade and autophagy in cancer therapy. Onco Targets Ther 2017;10:1803-7.

12. Zhang L, Wang J, Wei F, et al. Profiling the dynamic expression of checkpoint molecules on cytokine-induced killer cells from non-small-cell lung cancer patients. Oncotarget 2016;7:43604-15.

13. Kim D, Langmead B, Salzberg SL. HISAT: a fast spliced aligner with low memory requirements. Nat Methods 2015;12:357-60.

14. Jochems C, Hodge JW, Fantini M, et al. ADCC employing an NK cell line (haNK) expressing the high affinity CD16 allele with avelumab, an anti-PD-L1 antibody. Int J Cancer 2017;141:583-93.

15. Boyerinas B, Jochems C, Fantini M, et al. AntibodyDependent Cellular Cytotoxicity Activity of a Novel AntiPD-L1 Antibody Avelumab (MSB0010718C) on Human Tumor Cells. Cancer Immunol Res 2015;3:1148-57.

16. Benson DM Jr, Bakan CE, Mishra A, et al. The PD-1/PDL1 axis modulates the natural killer cell versus multiple myeloma effect: a therapeutic target for CT-011, a novel monoclonal anti-PD-1 antibody. Blood 2010;116:2286-94.

17. Khanna S, Thomas A, Abate-Daga D, et al. Malignant Mesothelioma Effusions Are Infiltrated by CD3(+) T Cells Highly Expressing PD-L1 and the PD-L1(+) Tumor Cells within These Effusions Are Susceptible to ADCC by the Anti-PD-L1 Antibody Avelumab. J Thorac Oncol 2016;11:1993-2005.

18. Mesiano G, Todorovic M, Gammaitoni L, et al. Cytokineinduced killer (CIK) cells as feasible and effective adoptive immunotherapy for the treatment of solid tumors. Expert Opin Biol Ther 2012;12:673-84.

19. Li J, Ye L, Shi X, et al. Repulsive guidance molecule $\mathrm{B}$ inhibits metastasis and is associated with decreased mortality in non-small cell lung cancer. Oncotarget 2016;7:15678-89. 
20. Li J, Chen Y, Shi X, et al. A systematic and genomewide correlation meta-analysis of PD-L1 expression and targetable NSCLC driver genes. J Thorac Dis 2017;9:2560-71.

21. Fujii R, Friedman ER, Richards J, et al. Enhanced killing of chordoma cells by antibody-dependent cell-mediated cytotoxicity employing the novel anti-PD-L1 antibody avelumab. Oncotarget 2016;7:33498-511.

22. Resch I, Shariat SF, Gust KM. PD-1 and PD-L1 inhibitors after platinum-based chemotherapy or in first-line therapy in cisplatin-ineligible patients: Dramatic improvement of

Cite this article as: Chen J, Chen Y, Feng F, Chen C, Zeng H, Wen S, Xu X, He J, Li J. Programmed cell death protein-1/ programmed death-ligand 1 blockade enhances the antitumor efficacy of adoptive cell therapy against non-small cell lung cancer. J Thorac Dis 2018;10(12):6711-6721. doi: 10.21037/ jtd.2018.10.111 prognosis and overall survival after decades of hopelessness in patients with metastatic urothelial cancer. Memo 2018;11:43-46.

23. Zhang Q, Liu XY, Zhang T, et al. The dual-functional capability of cytokine-induced killer cells and application in tumor immunology. Hum Immunol 2015;76:385-91.

24. Ferrari de Andrade L, Tay RE, Pan D, et al. Antibodymediated inhibition of MICA and MICB shedding promotes NK cell-driven tumor immunity. Science 2018;359:1537-42. 


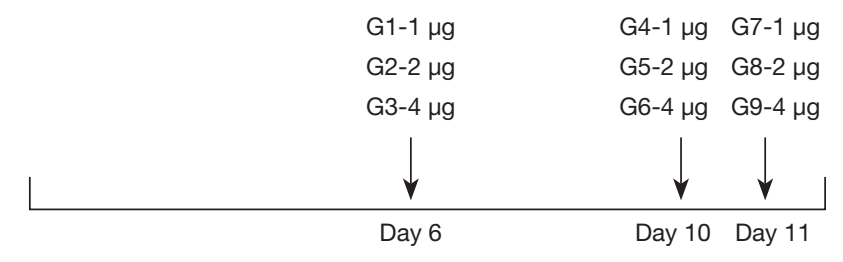

Figure S1 A timeline of the anti-PD-1 treatment of CIK cells cultures. PD-1, programmed cell death protein-1; CIK, cytokine-induced killer.

A

$\mathrm{CIK}+\mathrm{PD}-1$
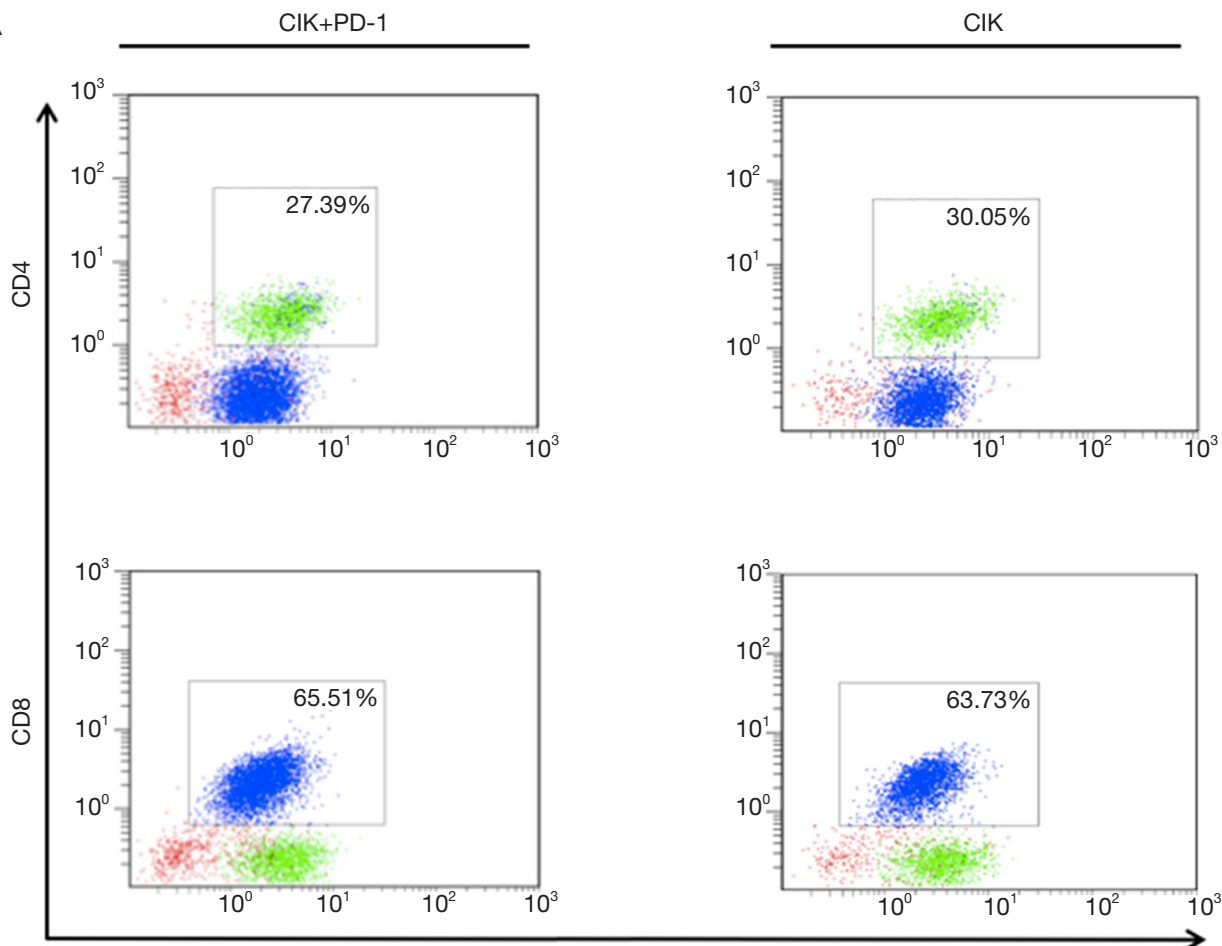

CD3

B

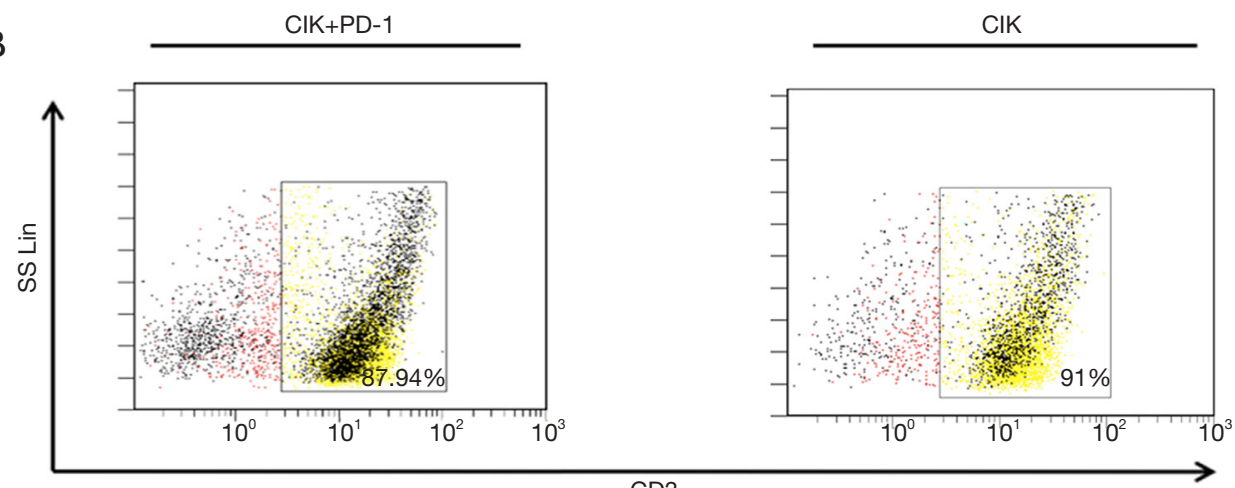

CD3
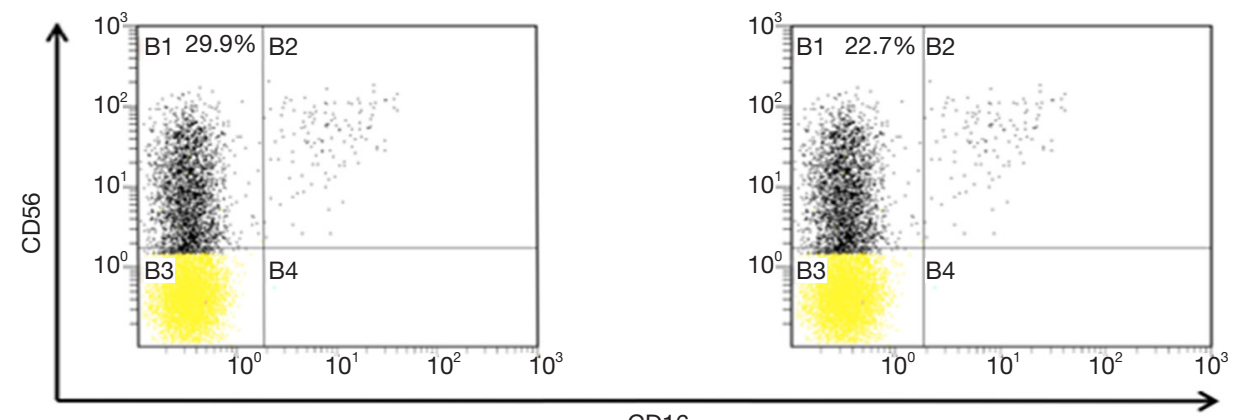

CD16
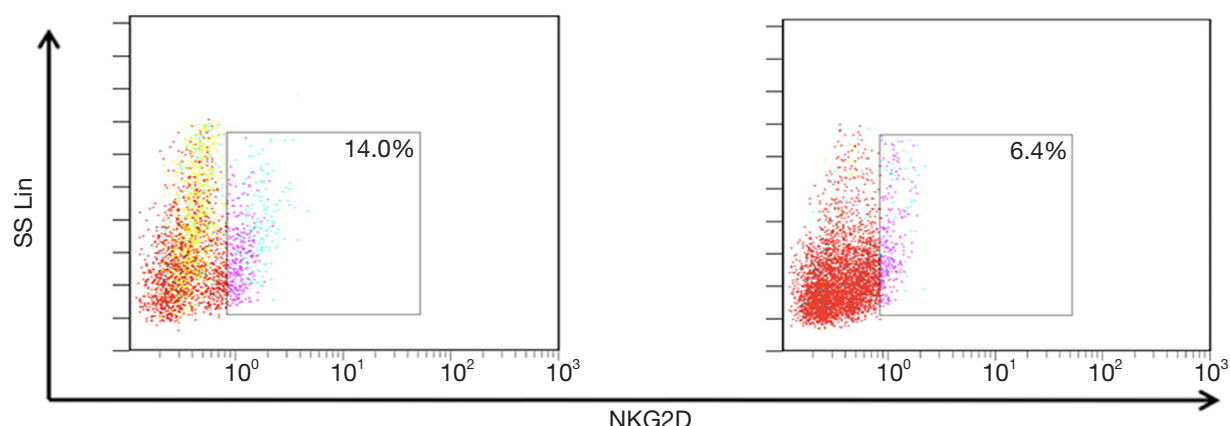

Figure S2 Molecular characterization of CIK cells co-cultured with the PD-1 antibody. The phenotypes of the CIK (cultured with or without the PD-1 mAb) used to target $\mathrm{H} 1975$ for 6 days. The proportions of $\mathrm{CD}^{+}$and $\mathrm{CD}^{+}$cells (A). The proportions of CD56 $6^{+}$cells gated from the $\mathrm{CD}^{+}$population, and the expression of NKG2D (B). CIK, cytokine-induced killer; PD-1, programmed cell death protein-1; NKG2D, natural killer group 2D.

$\begin{array}{ll}\mathrm{G} 1-1 \mu \mathrm{g} & \mathrm{G} 4-1 \mu \mathrm{g} \mathrm{G} \text { G- } 1 \mu \mathrm{g} \\ \mathrm{G} 2-2.5 \mu \mathrm{g} & \mathrm{G} 5-2.5 \mu \mathrm{g} \mathrm{G8}-2.5 \mu \mathrm{g} \\ \mathrm{G} 3-5 \mu \mathrm{g} & \mathrm{G} 6-5 \mu \mathrm{g} \text { G9-5 } \mu \mathrm{g} \\ \downarrow & \downarrow \\ \text { Day } 11 & \text { Day 12 Day 13 }\end{array}$

Figure S3 A timeline of the anti-PD-L1 treatment of NK cells cultures. PD-1, programmed cell death protein-1; NK, natural killer. 